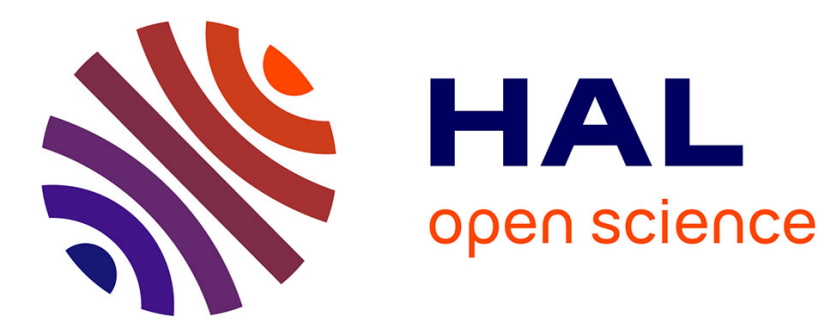

\title{
On the variation of interaural time differences with frequency
}

\author{
Victor Benichoux, Marc Rebillat, Romain Brette
}

\section{To cite this version:}

Victor Benichoux, Marc Rebillat, Romain Brette. On the variation of interaural time differences with frequency. Journal of the Acoustical Society of America, 2016, 139 (4), pp.1810-1821. 10.1121/1.4944638 . hal-01302795

\section{HAL Id: hal-01302795 \\ https://hal.science/hal-01302795}

Submitted on 15 Apr 2016

HAL is a multi-disciplinary open access archive for the deposit and dissemination of scientific research documents, whether they are published or not. The documents may come from teaching and research institutions in France or abroad, or from public or private research centers.
L'archive ouverte pluridisciplinaire HAL, est destinée au dépôt et à la diffusion de documents scientifiques de niveau recherche, publiés ou non, émanant des établissements d'enseignement et de recherche français ou étrangers, des laboratoires publics ou privés. 


\begin{tabular}{|c|c|}
\hline 2 & Victor Benichoux ${ }^{\mathrm{a}) \mathrm{b}}$ ) and Romain Brette ${ }^{\mathrm{a})}$ \\
\hline 3 & Institut de la Vision, \\
\hline 4 & (INSERM U968, \\
\hline 5 & CNRS UMR 7210, \\
\hline 6 & UMR S 968) \\
\hline 7 & 17 \\
\hline 8 & rue Moreau \\
\hline 9 & 75012 Paris, \\
\hline 10 & France \\
\hline 11 & Marc Rébillat \\
\hline 12 & PIMM \\
\hline 13 & Arts et Métiers ParisTech - CNRS - CNAM, \\
\hline 14 & 151 \\
\hline 15 & Boulevard de l'Hopital \\
\hline 16 & 75013 Paris, \\
\hline 17 & France \\
\hline
\end{tabular}

18

(Dated: February 15, 2016)

a) Also at: Institut d'Etudes de la Cognition, Ecole Normale Supérieure, Paris, France Frequency dependence of Interaural Time Differences 1 


\begin{abstract}
Interaural time difference (ITD) is a major cue to sound localization in humans and animals. For a given subject and position in space, ITD depends on frequency. This variation is analyzed here using an HRTF database collected from the literature and comprising human HRTFs from 130 subjects and animal HRTFs from six specimens of different species. For humans, the ITD is found to vary with frequency, in a way that shows consistent differences with respect to a spherical head model. Maximal ITD values were found to be about $800 \mu$ s in low frequencies and $600 \mu \mathrm{s}$ in high frequencies. The ITD variation with frequency (up to $200 \mu$ s for some positions) occurs within the frequency range where ITD is used to judge the lateral position of a sound source. In addition, ITD varies substantially within the bandwidth of a single auditory filter, leading to systematic differences between envelope and fine-structure ITDs. Because the frequency-dependent pattern of ITD does not display spherical symmetries, it potentially provides cues to elevation and resolves front/back confusion. The fact that the relation between position and ITDs strongly depends on the sound's spectrum in turn suggests that humans and animals make use of this relationship for the localization of sounds.
\end{abstract}

PACS numbers: 4366Qp, 4366Pn, 4380Lb 


\section{INTRODUCTION}

21 In humans and many animals, a major cue to localize sounds in the horizontal plane is

22 the difference in time between the peaks and valleys of the acoustical wave at the two ears, i.e.

23 the interaural time difference (ITD). Remarkably, humans can distinguish ITD differences

24 as low as $20 \mu$ s for a wide array of sound spectra and envelope characteristics below about

$251.5 \mathrm{kHz}$ (Mills, 1958; Brughera et al., 2013). Furthermore, ITD has been shown to have a

26 stronger influence than interaural level differences (ILD) on the perceived lateral location of

27 sounds with energy in low frequencies (below 2.5kHz, (Wightman and Kistler, 1992)). In

28 animals as well, ITD is used as a cue to sound location: cats, gerbils, birds, reptiles and

29 others have dedicated neural structures in the auditory brainstem to process ITD (Grothe

30 et al., 2010). Understanding the neural processing of ITDs requires a precise knowledge of

31 the nature of the temporal disparities imposed by the head, body and environment.

32 The relationship between source position and ITD is constrained by morphological pa-

33 rameters including the interaural distance, head, ear position, shape, torso, and (even)

34 hair (Duda et al., 1999; Algazi et al., 2001b). Measuring ITDs for tones using a manikin,

35 Kuhn (1997) found that the ITD also varies systematically with the frequency of the tone,

36 as reproduced in Fig. 1a. The ITD for a $2000 \mathrm{~Hz}$ tone presented at $45^{\circ}$ is $400 \mu \mathrm{s}$ (Fig. 1a,

37 dashed line), while the ITD for a $500 \mathrm{~Hz}$ tone at the same position is $600 \mu \mathrm{s}$ - about

$3850 \%$ larger. Conversely, sounds presented at different positions can produce the same ITD,

39 provided they have different frequency contents. It follows then that spatial position cannot

40 be estimated from ITD independently of sound frequency. This physical phenomenon is

41 known, and has been observed in models where the head is a rigid sphere (Kuhn, 1977),

42 or an ellipsoid (Cai et al., 2015). As an illustration we computed ITDs from the spherical

43 head model (details below) using a head radius of $9.3 \mathrm{~cm}$ (as reported in (Kuhn, 1977)), and

44 plotted it on Fig. 1b.

45 Despite these early insights, the dependence of ITD on frequency in humans and animals

46 has not, to our knowledge, been quantitatively examined. We propose here to bridge that

b) Electronic address: victor. benichoux@ucdenver.edu 
47 gap by a careful and in-depth assessment of the frequency-dependence of ITD in human and 48 animal acoustical data. Furthermore, we provide a new interpretation of this dependence, 49 showing that it results in different ITDs in the envelope and fine-structure of auditory filters' 50 outputs.

51 After a review of the physics of the frequency-dependence of ITD using previous reports 52 of the spherical model (section II), we provide a quantitative analysis of this phenomenon 53 in acoustical recordings of 130 subjects from four databases (section III). Further, we show 54 that ITDs between the envelopes and fine-structures of binaural signals are different (section 55 IV). Finally, we analyze the frequency-dependence of ITDs in the HRTFs of six different 56 animal species (section V), and show that the highly non-spherical nature of many animals' 57 heads makes it hard to predict the range of ITD from head size.
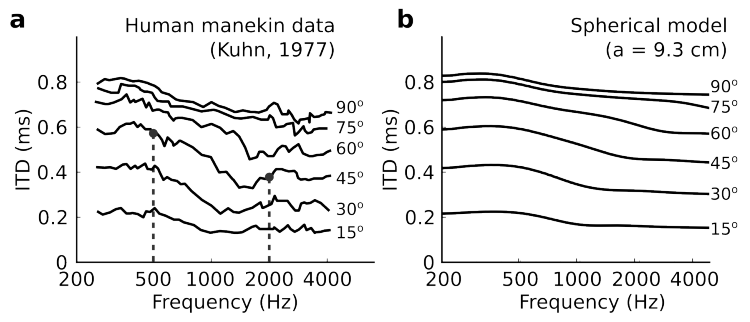

c
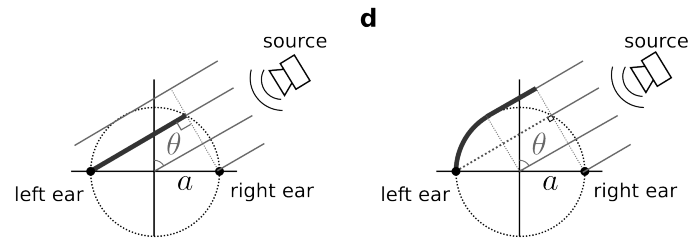

Figure 1: Frequency-dependence of ITD. (a) ITD measured with pure tones of varying frequency for different source positions on a human manikin (replotted from Kuhn (1977)). (b) ITD computed for a spherical head model with head radius $9.3 \mathrm{~cm}$. (c) Propagation of a planar sound wave with an acoustically transparent head. The additional pathlength to the contralateral ear (thick line) is a sine function of the azimuth angle $\theta$. (d) Propagation of a high frequence planar sound wave diffracted by a sphere. The additional path to the contralateral ear is the thick line. 


\section{II. SCATTERING AND FREQUENCY-DEPENDENT ITDS}

\section{A. Phase ITD}

The complete acoustical transformation occurring between a point source and the ears of a subject can be modeled as a pair of filters $\left(H_{L}, H_{R}\right)$ usually termed Head Related Transfer Functions (HRTFs). The phase ITD (ITD , as reported in the original (Kuhn, 1977) study), is the ITD of the fine-structure of the binaural signal, defined at any frequency $f$ using the phase response of the HRTFs:

$$
\operatorname{ITD}_{\mathrm{p}}(f, \theta, \phi)=\frac{1}{2 \pi f} \angle\left[\frac{H_{L}(f, \theta, \phi)}{H_{R}(f, \theta, \phi)}\right]
$$

60 where $\theta$ is azimuth measured in radians, $\phi$ is elevation (in standard vertical-polar coordinates:

61 azimuth between $-180^{\circ}$ and $180^{\circ}$, and elevation between $-90^{\circ}$ (down) and $90^{\circ}$ (up)). The

62 bracket operator $\angle$ is the unwrapped phase operator that yields a continuous phase spectrum

63 (not constrained to $[-\pi, \pi]$ ). By convention, positive azimuth values indicate that the source

64 is to the right of the subject, where $\mathrm{ITD}_{\mathrm{p}}$ is positive.

\section{B. ITD in the spherical head model}

In a first, simplified geometrical model of ITD, we can consider a planar acoustical 67 wave incident on an acoustically transparent head (Figure 1c). In this case, the ITD is

68 the difference in path lengths to the two ears (thick line) divided by the speed of sound: $69 \operatorname{ITD}(\theta)=2 \frac{a}{c} \sin (\theta)$, where $\theta$ is the azimuth of the sound source, $c$ the speed of sound in air 70 and $a$ the head radius. In this description, ITD does not depend on frequency: ITD does

71 show frequency dependence because the head is not acoustically transparent.

72 A more plausible acoustical model of the situation is to assume that the head is a rigid 73 sphere, with ears lying on a diameter. The first observation that ITD is frequency-dependent 74 in this context is attributed to Lord Rayleigh's spherical head model (Rayleigh and Lodge, 75 1904). Many studies have then used this model to analyze binaural cues (Duda and Martens, 76 1998; Kuhn, 1977). In particular, the phase ITD, i.e. ITD $_{\mathrm{p}}$ as defined in Eq. 1, can be 
77 numerically calculated at all frequencies for the spherical head model, as shown on Fig. 1b

78 (for sources at infinite distance from the head, following Bruneau (2010)). In this model,

$79 \mathrm{ITD}_{\mathrm{p}}$ for any given source position generally decreases with increasing frequency (Fig. 1b),

80 which is broadly consistent with the human manikin data reported by Kuhn (1977) and

81 shown in Fig. 1a.

For high frequencies, when the wavelength is small compared to the head radius, the ITD tends to the difference between the shortest path lengths to the two ears divided by the speed of sound (thick line in Figure 1c), which is expressed in Woodworth's formula:

$$
\operatorname{ITD}_{\mathrm{HF}}(\theta)=\frac{a}{c}(\sin (\theta)+\theta)
$$

The low-frequency limit of ITD can be calculated by considering the first terms in the spherical-harmonic development of the acoustical field solution (Kuhn, 1977):

$$
\operatorname{ITD}_{\mathrm{LF}}(\theta) \approx 3 \frac{a}{c} \sin (\theta)
$$

82 The ratio between low and high frequency ITD is then: $\frac{3 \sin (\theta)}{\theta+\sin \theta}$, which is always greater than 83 one. Thus the the low-frequency ITD is always greater than the high frequency ITD. For 84 azimuths $\theta$ between 0 and $\pi / 2$ radians $\left(90^{\circ}\right)$, this ratio is a monotonically decreasing function 85 of $\theta$. For sources near $0^{\circ}$, the ITD is $50 \%$ larger at high frequency relative to low frequency, 86 but for those positions (close to the midline) ITD values are close to zero. Conversely, when 87 the ITD is maximal for azimuths near $90^{\circ}$, the low frequency ITD is only about $16 \%$ larger 88 than the high frequency ITD. Readers should note that this is hard to see on $1 \mathrm{~b}$.

\section{Visualization of the scattering phenomenon}

At low frequency, the head is small compared the wavelength of the sound, and one

91 might infer that the ITD should be close to the situation when the head is acoustically 92 transparent (Fig. 1c). This would predict a smaller ITD than in high frequency, yet the 93 opposite occurs. The reason for this counter-intuitive phenomenon is that the sphere is not 94 an obstacle between the source and the ears, but rather the ears are on the sphere and 95 diffraction phenomena are at play (Kuhn, 1977). 


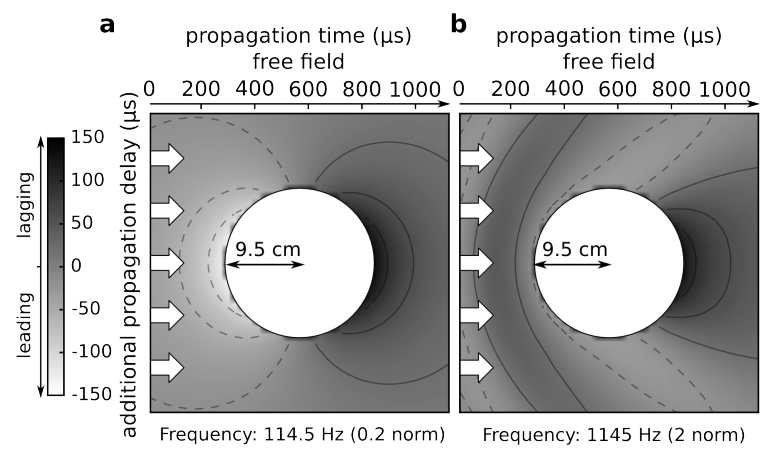

Figure 2: Propagation time of a planar sound wave in the presence of a sphere, relative to the propagation time in free field, for tone frequencies $114.5 \mathrm{~Hz}$ (a) and $1145 \mathrm{~Hz}$ (b). Propagation time in free field (no head) is shown on top. Negative values (lighter shades) indicate regions where phase is leading, and positive values (darker shades) indicate regions where the sound phase is lagging.

To get a better grasp of the situation, we calculated and represented the acoustical field 97 in the vicinity of the head, using the formula in Bruneau (2010, paragraph 5.2.3). In Fig. 2, 98 we show the steady-state propagation time of a monochromatic planar wave emanating 99 from an infinite-distance source to the left $\left(\theta=90^{\circ}\right)$, relative to the propagation time for 100 an acoustically transparent head (free field). For head radius of $9.5 \mathrm{~cm}$, the free field ITD 101 is about $550 \mu \mathrm{s}$. For a low frequency tone (about $115 \mathrm{~Hz}$, Fig. 2a), a phase lead appears on 102 the ipsilateral side of the head (the acoustical wave is "compressed" against the head), and 103 a phase lag appears on the contralateral side (the wave must turn around the head). As a 104 result, the ITD is $550+150+150=850 \mu$ s. For a high-frequency tone $(1145 \mathrm{~Hz}$, Fig. 2 b), 105 the propagation time to the ipsilateral ear (left) is not affected by the head but there is 106 still a phase lag at the contralateral ear, corresponding to the additional path length. As a 107 result, the ITD is $550+150=700 \mu$ s, which is smaller than at low frequency.

108 The physical phenomenon is entirely specified by the wavelength $(\lambda=c / f)$ of the 109 acoustical wave relative to the size of the sphere. To account for the effect of the size of the 110 head, it is thus convenient to introduce a normalized frequency scale, where unit normalized 
111 frequency $\left(f_{\text {norm }}=1\right)$ corresponds to the physical frequency of a wave with wavelength 112 equal to one sphere circumference: $f=c / 2 \pi a$. Scaling head size also scales ITD (it is 113 inversely related), and therefore ITD can also be normalized, so that a scaled ITD of unity $114\left(I T D_{n}=1\right)$ corresponds to a physical ITD of $a / c$, the time for sound to propagate one

115 radius of the spherical head. Assuming a head radius of $a=9.5 \mathrm{~cm}$, the low-frequency 116 condition of Fig. 2 corresponds to a normalized frequency of 0.2 , and the high-frequency 117 condition to a normalized frequency of 2.0 .

118 From this description, in terms of normalized time and frequency, the effect of changing 119 the head size on $\operatorname{ITD}_{\mathrm{p}}(f)$ is easier to grasp. It is two-fold: for a given normalized frequency, 120 the ITD depends linearly on head size; and the frequency scale on which ITD varies depends 121 linearly on the inverse of head size. In particular, the transition between the low and high 122 frequency regimes occurs at lower frequencies for bigger head sizes.

\section{III. FREQUENCY-DEPENDENCE OF ITD IN HUMAN HRTFS}

Human head morphology is more complex than a sphere, and other parts of the human 125 body also influence the ITD (Kuhn, 1977). Furthermore, comparing the human-manikin 126 ITDs to the spherical-head ITDs in Fig. 1a-b reveals that ITDs exhibit a more complex 127 frequency-dependence in humans than in the spherical head model. Therefore it is necessary 128 to analyze the frequency-dependence of ITD from HRTF measured in real human subjects.

\section{A. HRTF databases}

HRTF data were obtained from three publicly available databases (ARI (ARI, 2010),

131 CIPIC (Algazi et al., 2001a) and LISTEN-V1 (Warusfel, 2002)). Another set of data was 132 specifically recorded for this project (LISTEN-V2), following the protocol of the LISTEN-V1 133 database, and has not been made public yet. All data are available from the corresponding 134 author on request. Overall, this combined dataset includes 130 subjects (Table I). Because 135 these databases were obtained in slightly different conditions (in particular spatial measure- 
136 ment grids and number of samples), ITDs were evaluated separately in each database, and

137 then all statistics were interpolated on a common space-frequency grid (that of LISTEN-V2)

138 using a natural neighbor interpolation. Results are therefore always presented with a spatial

139 resolution of $5^{\circ}$ in azimuth and about $15^{\circ}$ elevation.

140 B. Frequency dependence of human ITDs

141 1. Acoustical head radius estimation and normalization

142 As discussed above, the subject's head size affects ITD across positions and frequencies

143 in a way that is completely predicted by the acoustics of sound propagation. We are not,

144 however, interested in the variability of ITD cues across the population of subjects that

145 is explained by head size. Rather, we are interested in how this variability reflects the

146 variability in head morphologies. Furthermore, increasing headsize systematically shrinks

147 the frequency axis, thus averaging different subjects' ITD at each frequency will spuriously

148 smooth out ITD variations. Therefore, in order to account for the effect of head size across

149 the population of subjects, we normalize the time and frequency axes of each subject's

150 ITD data using a measure of the head of the subject derived from its HRTFs (usually

151 termed acoustical head radius, e.g. in (Algazi et al., 2001b)). Similar normalization methods

152 have been proposed in the context of reducing variability in spectral notches position and 153 amplitude (Middlebrooks, 1999).

154 We define the "acoustical head radius" for each subject as the radius of the spherical 155 model which best matches the subject's high-frequency ITD. More precisely, for each subject

156 we compute the high frequency ITD at all positions as the average phase ITD between $3 \mathrm{kHz}$

157 and $5 \mathrm{kHz}$. We compute the spherical-model high-frequency ITDs in the exact same way 158 and for the same positions. The radius of the sphere is then adjusted so as to minimize the 159 squared differences between the subject's ITDs and the sphere's. This regression is performed 160 using a standard gradient-descent algorithm. The best-fitting sphere radius resulting from 161 this procedure is the acoustical head radius of the subject. We validated this method by 
162 simulating HRTFs using the spherical head model, and recovering the radius of the simulated 163 spherical head. As expected, we found that this method estimated accurately the radius of 164 the sphere within $0.01 \mathrm{~mm}$ (over a range of sphere radii from $5 \mathrm{~cm}$ to $15 \mathrm{~cm}$ ).

165 We then estimated the acoustical head radius of each subject in the population. We 166 found the average head radius over all subjects to be $9.5 \mathrm{~cm}(\mathrm{~N}=130, \pm 0.48 \mathrm{~cm}$ standard 167 deviation, 8.3 to $10.8 \mathrm{~cm}$ range). For each subject, we computed the mean squared error 168 between ITDs derived from the optimal spherical model and acoustically measured ITDs.

169 The mean squared error was on average $67 \mu \mathrm{s} \pm 22 \mu \mathrm{s}$ STD across the population, indicative 170 of a consistently good fit. The average head radius value we found is slightly higher than 171 the one reported in the (Algazi et al., 2001b) study, which was obtained using another ITD 172 estimation method (onset-time differences), and only on positions on the horizontal plane. 173 The same estimation and fitting method on our data yields a radius of $8.43 \mathrm{~cm}( \pm 1.14 \mathrm{~cm})$, 174 consistent with the value reported by (Algazi et al., 2001b).

175 The goal of this normalization procedure was to account for the systematic effect of 176 head size on the variability of the ITD measures across the population. We computed the 177 standard deviation of ITD at each frequency and position across the population of subjects 178 before and after normalization. The average standard deviation before normalization was 54 $179 \mu$ s and reduced by about $20 \%$ with normalization (42 $\mu$ S STD, using the average head size 180 of $9.5 \mathrm{~cm}$ to convert to physical units). This reduction was quantitatively different across 181 databases, and more pronounced in databases with more subjects (45\% in LISTEN-V2).

182 Most ITDs and derived statistics are reported in the rest of the manuscript on normalized 183 frequency and ITD scales with a single pair of normalization factors (frequency and ITD) 184 for the population. For ease of reading, when representing human ITD data, ITDs and 185 frequencies are represented both in normalized and direct physical units, with a conversion 186 factor assuming a head radius equal to the average over the population $(a=9.5 \mathrm{~cm})$. For 187 this value of the head radius, a normalized frequency of 1 corresponds to a frequency of 573 $188 \mathrm{~Hz}$, and a normalized ITD of 1 corresponds to an ITD of $278 \mu \mathrm{s}$. Therefore, to convert from 189 normalized to physical ITD in $\mu \mathrm{s}$, multiply the normalized value by $278 \mu \mathrm{s}$. To convert from 


\section{Asymptotic ITD in the horizontal plane}

192

193

194

195

196

197

198

199

200

201

202

203

204

205

206

207

208

209

210

211

212

We estimated the asymptotic low and high frequency values of the ITD to compare them with theoretical predictions from the spherical model (Figure 3).

The high-frequency $\operatorname{ITD}, \operatorname{ITD}_{\mathrm{HF}}(\theta)$ is estimated as the mean ITD between $f_{\text {norm }}=7$ $(4010 \mathrm{~Hz})$ and $f_{\text {norm }}=8(4600 \mathrm{~Hz})$. As per construction, the Woodworth formula (Figure $3 \mathrm{a}$, black) fits the data (shaded area: mean $\operatorname{ITD}_{\mathrm{HF}}(\theta)$ over subjects $\pm 1 / 2$ s.d.), except when the azimuth is in the $70-110^{\circ}$ range, which has been previously documented (Aaronson and Hartmann, 2014). Figure 3B shows the low frequency prediction with Kuhn's formula (Eq. 3) alongside mean ITD estimated from HRTFs between $f_{\text {norm }}=0.5(290 \mathrm{~Hz})$ and $f_{\text {norm }}=0.6(350 \mathrm{~Hz})$, termed low frequency $\operatorname{ITD}_{\mathrm{LF}}(\theta)$. Consistent with previous reports, the approximation of the low-frequency ITD is reliable.

On Figure 3c, the values of ITD across frequency are reported for seven positions on the horizontal plane $\left(0,30,60,90^{\circ}\right.$ and the symmetrical positions). Curves represent the average normalized ITD across subjects, and shaded areas are \pm 1 s.d.. The standard deviation of the normalized ITDs at each frequency and position was on average $46 \mu \mathrm{s}$ (0.16 normalized). This variability is relatively small: it is about $6 \%$ of the maximal ITD value observed, and is about a factor of two larger than a human just-noticeable difference (JND) in ITD (the smallest ITD difference perceptible by human subjects (Mills, 1958)).

In addition, the plots on Figure 3c reveal some fine variations of ITD with frequency that are not accounted for by the spherical model (e.g. the low frequency bump for azimuth $\pm 30^{\circ}$ ). This reveals that deviations of the human head morphology from a sphere contribute systematically to the ITD versus frequency relationship. 

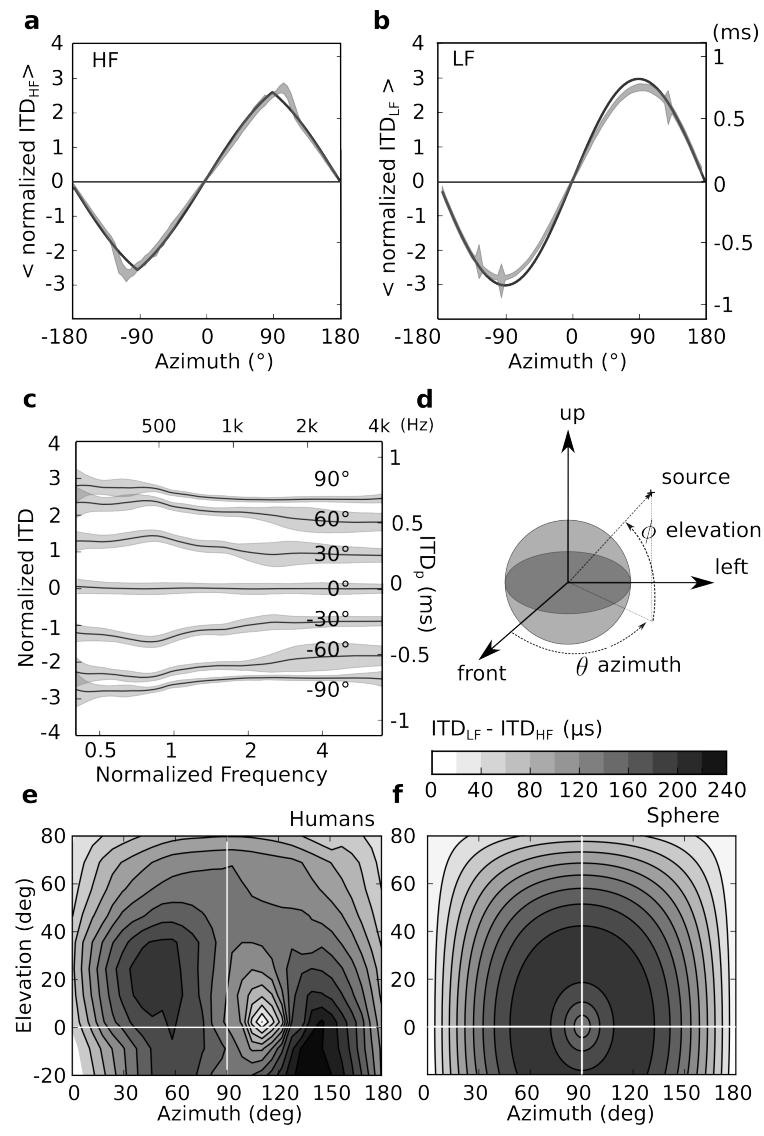

Figure 3: Frequency dependence of ITD in human subjects. (a) Inter-individual average normalized ITD in high frequency as a function of azimuth $\pm 1 / 2$ s.d. (shaded area). Black line indicates the theoretical value from the Woodworth model (Eq. 2). Corresponding ITD values for a head radius of $9.5 \mathrm{~cm}$ are shown on the right of panel b. (b) Average normalized ITD in low frequency $\pm 1 / 2$ s.d. (shaded area), black line indicates the theoretical value from Kuhn's formula (Eq. 3). (c) Average normalized ITD (black lines) as a function of frequency for seven source positions (shaded area: $\pm 1 / 2$ s.d.). (d) Azimuth $\theta$ and elevation $\phi$ are defined in a standard vertical-polar coordinate system (see text). (e) Difference between high- and low-frequency normalized ITD as a function of elevation and azimuth. Physical ITD is calculated for a head radius of $9.5 \mathrm{~cm}$. (f) Same as (e) for the spherical model. Normalized units correspond to a head radius of $9.5 \mathrm{~cm}$. 


\section{Frequency variation of ITD as a function of azimuth and elevation}

214 Consistent with previous reports, our data show that ITD is frequency-dependent in

215 human HRTF, with similar differences between low and high frequency values as in the 216 spherical models in the horizontal plane. We now quantify this difference as a function 217 of both azimuth $\theta$ and elevation $\phi$. Recall that we used a vertical-polar coordinate system 218 (Fig. 3d). Fig. 3e shows the average difference ITD $_{\mathrm{LF}}-\mathrm{ITD}_{\mathrm{HF}}$ across subjects for all positions 219 on the spatial grid, converted to physical ITD values assuming head radius of $9.5 \mathrm{~cm}$. For 220 comparison, the same quantity is shown for the sphere on Fig. 3f.

221 The difference between high and low frequency ITD exceeds $50 \mu$ s for most of the 222 positions on the sphere, and can reach more than $200 \mu \mathrm{s}$. As a comparison, human subjects 223 can discriminate ITDs differing by a JND of only $20 \mu$ s (Mills, 1958). Therefore, the variation

224 of ITD with frequency should be perceptually significant for most source positions away from 225 the midline.

226 For large enough source distances, the pressure at any point on the surface of a sphere 227 depends only on the angle between the ray from the center of the sphere to the source, 228 and the ray to the measurement point on the surface of the sphere (Duda and Martens, 229 1998). Because of this symmetry property, binaural cues are constant for sources lying on 230 so-called cones of confusion, centered on the interaural axis. In other words, acoustical cues 231 in a spherical head model only depend on the angle of the source acoustical wave and the 232 medial-sagittal plane: the incidence angle $\beta=\arcsin (\cos (\phi) \sin (\theta))$. Cones of confusion are

233 then the set of points of equal incidence angle $\beta$. This aspect makes it hard to differentiate 234 sound sources positioned symmetric to the interaural axis, which includes front and back 235 positions.

236 Consistent with previous reports in humans, our data show that cones of confusion are 237 centered around source positions directly facing the ears (that is, at the same azimuth and 238 elevation as the ears, Aaronson and Hartmann, 2014). Furthermore, they appear distorted 239 (around $(\theta, \phi)=\left(110^{\circ}, 5^{\circ}\right)$, Fig. 3e). In particular, the variation of ITD with frequency 
quantitatively differs between front and back (and up and down) positions.

241

\section{Maximal ITD}
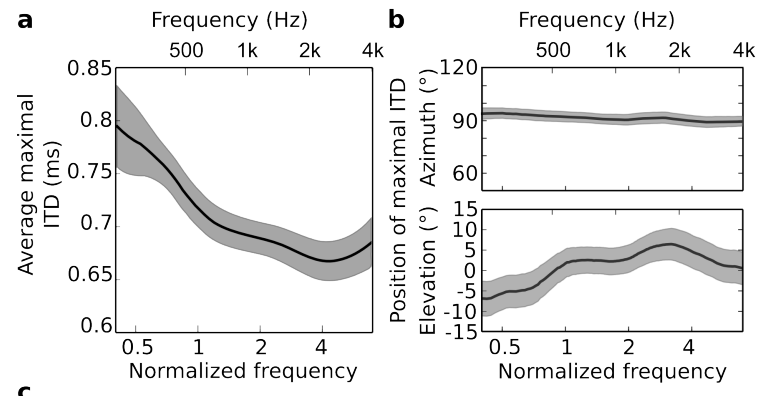

C

$$
\begin{array}{llllllll}
0.5 & 0.75 & 1 & 1.25 & 1.5 & 1.75 & 2 & (\mathrm{kHz})
\end{array}
$$
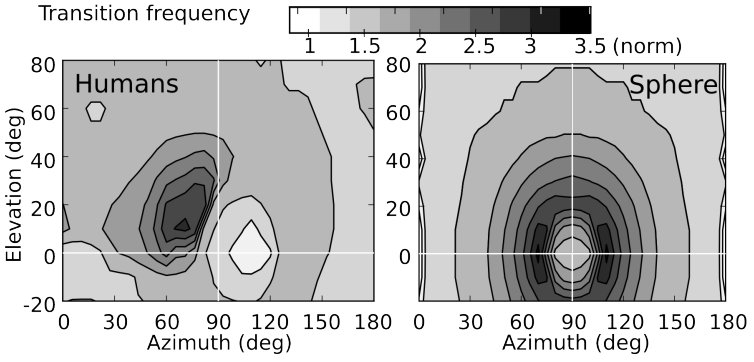

Figure 4: Maximal ITD and transition frequency in human subjects. (a) Maximal ITD across subjects as a function of frequency. (b) Azimuth (top) and elevation (bottom) where ITD is maximal as a function of frequency Shaded areas of (a) and (b) are the mean $\pm 1 / 2$ s.d. (c) Transition frequency (see text) as a function of azimuth and elevation in humans (left) and in the spherical model (right). Normalized units converted assuming a head radius of $9.5 \mathrm{~cm}$.

We estimated, from the unnormalized data, the maximal ITD over positions for each

243 frequency and subject (reported here on a normalized frequency scale). By nature, ITD 244 estimation can be unstable in high frequency because of the ambiguity inherent when un245 wrapping a phase response, and in low frequency because of the low frequency resolution. 246 This is especially prominent when automatically processing a large number of recordings 247 (several hundred positions, and subjects), and creates many outlier datapoints, which posi248 tively biases the estimation of a maximal ITD. 

maximum, which we define here as the maximal ITD. Consistent with the theory and previ-

251 ous reports, we found that the maximal low-frequency ITD value is $813 \mu \mathrm{s} \pm 70 \mu$ s (s.d., see 252 Fig. 4a). The maximal broadband ITD - computed as the peak lag of the cross-correlated 253 impulse responses, was found to be $612 \mu \mathrm{s} \pm 34 \mu$ s (s.d.). This value is very close to the 254 value of the maximal high frequency ITD, $688 \mu \mathrm{s} \pm 47 \mu$ s (s.d., see Fig. 3b).

255 The maximal ITD occurs for azimuth around $95^{\circ}$ (Fig. 4b), for which the source is 256 directly facing one of the two ears, which is consistent with previous reports (Aaronson and 257 Hartmann, 2014). There are systematic variations of the position of maximal ITD with 258 frequency, but it remains near eccentric azimuths $\left( \pm 90^{\circ}\right)$ and close to the horizontal plane $259\left(-10^{\circ}\right.$ to $\left.10^{\circ}\right)$. In the spherical model computations, the maximal ITD is reached at $\theta=90^{\circ}$ 260 (Eq. 3), in humans it occurs for positions slightly more to the back (Fig. 4b), and for 261 sources originating from below or above the horizontal plane, depending on the frequency 262 of the signal (Fig. 4b).

\section{D. Transition between ITD regimes}

264 It could be argued that even though the ITD varies across frequency, this variation does 265 not occur in the range where ITD is used as a cue to azimuth (i.e. the ITD is constant 266 below $1.8 \mathrm{kHz}$ ). To assess this, we examined the shape of the ITD versus frequency curves, 267 specifically trying to get at the frequency at which ITD effectively transitions between its low 268 and high frequency regimes. We define the transition frequency as the frequency at which 269 the ITD equals the average between its high frequency $\left(\operatorname{ITD}_{\mathrm{LF}}(\theta, \phi)\right)$ and low-frequency $270\left(\operatorname{ITD}_{\mathrm{HF}}(\theta, \phi)\right)$ values for a given position. Because in general ITD is a decreasing function of 271 frequency with a relatively narrow transition, this transition frequency allows us to separate 272 frequency regions of high and low ITD values for any position.

273 Figure $4 \mathrm{C}$ shows the transition frequency in humans and in the spherical head model 274 (for an infinitely distance source), as a function of azimuth and elevation. The transition 
275 frequency increases as the source is moving away from the median sagittal plane, up to an 276 azimuth angle $\simeq 70^{\circ}$ where a maximum value $f_{\text {tran }} \simeq 2.8$ (3.2 in the spherical model) is 277 reached. It then decreases until a minimum is reached at $\theta \simeq 110^{\circ}$ and $\phi \simeq 0^{\circ}\left(\theta=90^{\circ}\right.$ and $278 \phi=0^{\circ}$ in the spherical model).

279 In conclusion, for all positions, transition frequencies are between 1 and 3 (normalized 280 scale), which corresponds to physical frequencies between approximately 600 and $1700 \mathrm{~Hz}$. 281 The ITD thus varies substantially at frequencies within the range where ITD is the dom282 inant cue for sound laterality in the horizontal plane (Wightman and Kistler, 1992). The 283 magnitude of transition frequencies in humans is overall similar to the predictions of the 284 spherical model, yet as previously mentioned symmetries seen in the spherical model do not 285 appear in the human HRTF data.

\section{IV. ENVELOPE AND FINE-STRUCTURE ITD}

287 When a sound wave excites the cochleae, different points on the basillar membranes are 288 preferentially excited by energy in different frequency bands. Many neurons in the auditory 289 system, in particular neurons in the midbrain that are sensitive to ITD, are also tuned 290 to different frequencies and are tonotopically organized. We have shown that ITD varies 291 substantially across different frequency bands, that is, between distant auditory filters (Fig. 292 5a). An interesting question is whether ITD also varies substantially within a single auditory 293 filter (Fig. 5b), as it would then have direct physiological relevance. We relate this question 294 to the difference between envelope and fine-structure ITD.

\section{A. Variation of ITD within a auditory filter}

We first analyzed the variation of ITD within single channels for each position and 297 subject (same HRTF database as in Section III). The variation of ITD is defined as the 298 difference between the maximum and minimum $\mathrm{ITD}_{\mathrm{p}}$ in a frequency band with constant

$299 Q=4.3$ (one-third octave) or with equivalent-rectangular bandwidths (ERB) (Glasberg 

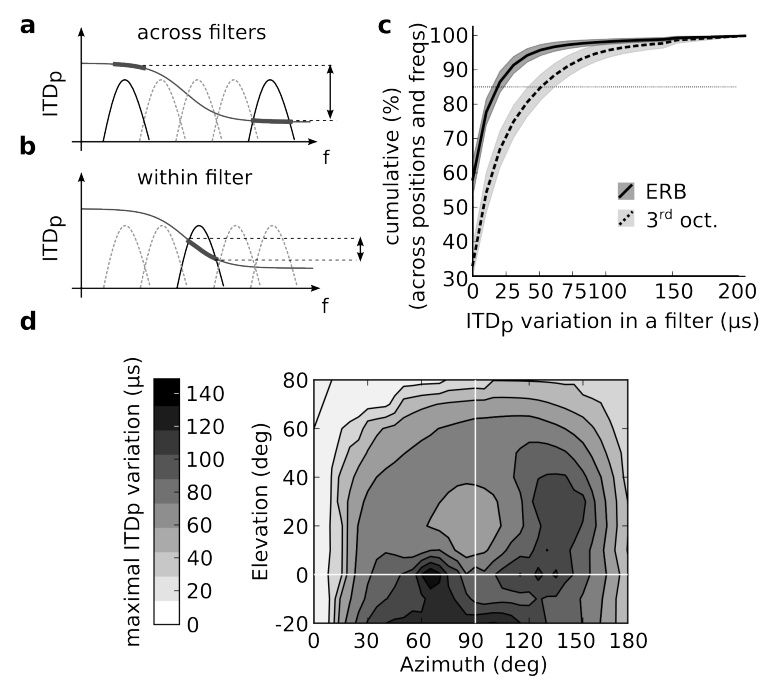

Figure 5: Variation of ITD within single auditory filters. (a) Schematics of the global variation of ITD across different auditory filters. (b) Schematics of the variation of ITD within a single auditory filter. (c) Proportion of positions and center frequencies where ITD variation within a single channel is smaller than a specified value. Lines are averages across population, \pm s.d.. Channel width is either 1 ERB or $1 / 3$ octave. (d) Maximal ITD variation within single channels, as a function of azimuth and elevation, with ERB-wide channels.

300 and Moore, 1990) (Fig. 5b). In the ERB scale, the Q factor value varies between 5 and 301 9, indicative of the relatively narrow band filtering imposed by the auditory periphery. 302 We computed the variation of ITD for center frequencies between 350 and $3000 \mathrm{~Hz}$ and for 303 positions close to the horizontal plane $\left(|\phi| \leq 20^{\circ}\right)$. We report the cumulative distributions of 304 ITD variation on Fig. 5c: curves display the percentage of positions and center frequencies 305 for which the ITD variation is lower than a given amount. In both conditions, for more 306 than $15 \%$ of the channels and positions the magnitude of the ITD variation is larger than $30725 \mu \mathrm{s}$ (Fig. 5c). In Fig. 5d, we show the maximal variation of ITD in single channels 308 as a function of azimuth and elevation (assuming ERB-wide channels). That is, for every 309 position, we report the variation of ITD in the channel where it varies the most. At specific 310 source positions, very large variations of ITD can occur within channels (up to $150 \mu \mathrm{s}$ ): the 
311 variation of ITD within a single channel is therefore quite substantial.

The fact that ITD varies within a frequency band means the signal undergoes more than a simple delay when passed through the HRTF. Mathematically, the phase responses of the monaural filters are nonlinear functions of frequency. We can approximate the IPD by an affine (i.e. linear with a non-zero intercept) function of frequency around the center frequency $f_{0}$ of a cochlear filter (Fig. 6a):

$$
\begin{aligned}
\operatorname{IPD}(f) & \stackrel{\text { def }}{=} \operatorname{ITD}_{p}(f) f \\
& \approx \operatorname{ITD}_{g}\left(f_{0}\right)\left(f-f_{0}\right)+\operatorname{IDI}\left(f_{0}\right)[1]
\end{aligned}
$$

312 where phases are expressed in cycle. The slope of this fit is the group ITD, which is the ITD 313 of the envelope (Marple Jr, 1999).

314 The intercept IDI is an additional shift in the phase of the fine-structure of the signal 315 (Fig. 6b). This shift only occurs when the phase ITD varies with frequency, i.e., when 316 propagation does not result in a pure delay. For this reason, we termed this binaural cue 317 the Interaural Diffraction Index (IDI, see Rebillat et al. (2014)). The IDI can be seen as a 318 measure of the difference between group and phase ITDs at any frequency, converted into a 319 phase value: IDI $=\left(\operatorname{ITD}_{p}-\operatorname{ITD}_{g}\right) f$. If IDI $=0$ cycles, phase and group ITD are equal, and 320 locally frequency-independent. When the IDI is positive, by convention the phase ITD has 321 a higher absolute value than the group ITD, and vice versa when IDI is negative.

\section{B. Estimating ITD in the envelope and fine-structure of binaural signals}

The group ITD is classically defined as the derivative of the unwrapped IPD curve with

324 respect to frequency as represented in Fig. 6a. Because of occasional errors of the phase 325 unwrapping operation, estimating the derivative from large sets of unwrapped IPD curve is 326 unreliable. Instead we use an equivalent approach wherein we perform circular-linear fits on 327 the wrapped IPD. The estimation can be formulated as a non linear least square problem, 328 where IDIand $\operatorname{ITD}_{\mathrm{g}}$ are chosen to minimize the fit error $\sum_{f}\left\|\operatorname{IPD}(f)-\left(\mathrm{IDI}+\operatorname{ITD}_{\mathrm{g}} f\right)\right\|^{2}$ over 329 a given frequency band, where the norm $\|$.$\| is a norm on phases. Because wrapped phase$ 


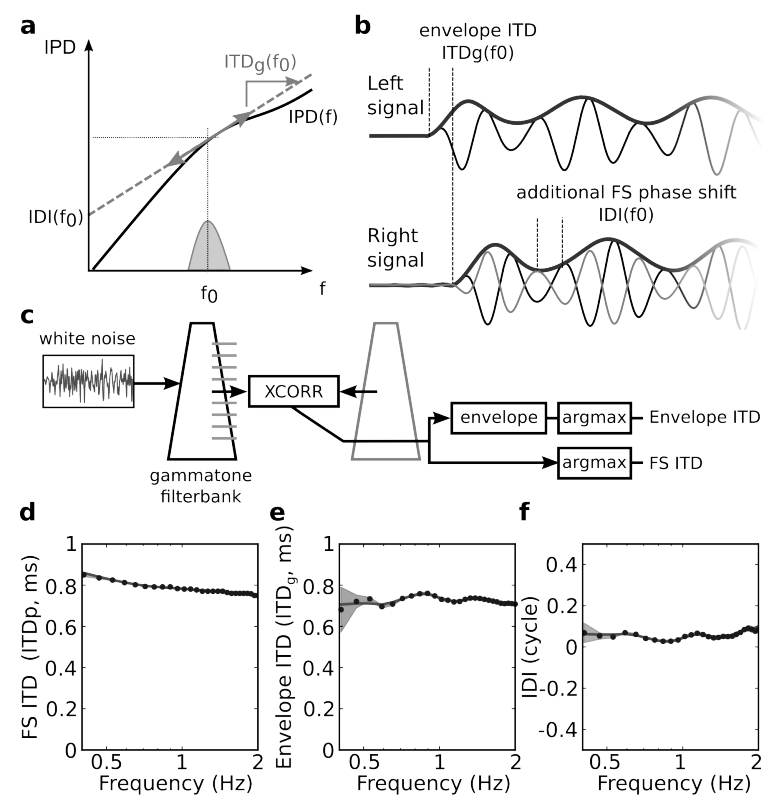

Figure 6: Envelope and fine-structure ITD. (a) The IPD for one position is unwrapped and an affine fit is taken locally around $f_{0}$. The intercept of the fit is the IDI and the slope the group ITD. (b) When the IDI is zero, the delay is frequency-independent and both envelope and fine-structure are delayed by the ITDg (bottom, black signal). When IDI is non zero, the fine-structure undergoes an additional phase shift equal to the IDI (bottom, grey signal). (c) Simulation: white noise is passed through HRTF filters for one position (spherical head model, azimuth $=70^{\circ}$ ). The resulting signals are then fed into gammatone filterbanks. The responses in the two banks are then cross-correlated, and the result is separated in envelope and fine-structure components. The time lag of the maximum of the cross-correlation is the phase ITD, and that of the maximum of the envelope of the cross-correlation is the envelope ITD (see text). (d) Results of estimating phase ITD, ITD $_{\mathrm{p}}$, from the IPD (plain line), and from simulations (mean: dots, shaded area: 95\% confidence interval). (e) Same as (d) for ITDg. (f) Same as (d) for IDI.

330 values are a circular quantity, so is the norm we use in the fit. It can be expressed as a cosine 331 function of its argument: $\|x\|^{2}=1-\cos (2 \pi x)$. The precise algorithm used is described in 332 more details in the Methods section of Luling et al. (2011). 
334 fine-structure of band-limited binaural signals, we simulated a simple model of the auditory 335 periphery. We then computed the envelope and fine-structure ITD in different frequency 336 bands of our auditory model (Fig. 6c) using a standard cross correlation approach. The 337 auditory periphery model consisted of two gammatone filter banks receiving 100-ms-duration 338 white-noise inputs convolved with the spherical HRTFs. Each filter's response was then 339 cross-correlated with the opposite frequency band. This operation is a good approximation 340 of the response of binaural neurons in the medial superior olive of mammals (Yin and Chan, 341 1990). The fine-structure ITD is obtained by computing the position of the maximum of 342 the cross correlation function. Then, the envelope of the cross-correlation is extracted using 343 a Hilbert transform, and the maximum computed. This procedure yields an estimate of 344 the delay in the envelopes (i.e. it is equivalent to computing the maximum of the cross345 correlation of the envelopes Marple Jr (1999)). The results of this simulation are plotted 346 on Fig. 6d-f. Dotted points (with $95 \%$ confidence intervals over repeated trials) represent 347 the ITDs estimated using the simplified auditory model. The theoretical predictions (plain 348 lines) were obtained by taking circular-linear fits of the IPD of the HRTF pair used. The 349 match between theory and simulation is excellent, which shows that the group ITD indeed 350 corresponds to the envelope ITD, which appears in the cross-correlation of the monaural 351 signals, and the IDI indeed corresponds to the additional shift of the fine-structure seen in 352 the cross-correlation function.

\section{C. Analysis of envelope and fine-structure ITDs in human HRTFs}

354 We computed ITDs in the envelope and fine-structure of the human data presented in 355 Section III, according to the methods presented above. On Figure 7 we present the average $356 \mathrm{ITD}_{\mathrm{p}}, \mathrm{ITD}_{\mathrm{g}}$ and IDI over the whole population. We observe that $\mathrm{ITD}_{\mathrm{p}}$ and $\mathrm{ITD}_{\mathrm{g}}$ can be 357 dramatically different in some frequency bands (typically around $1 \mathrm{kHz}$ ). As a result the IDI 358 is non-zero in that range (Figure 7e), which correponds to the frequency range just above 

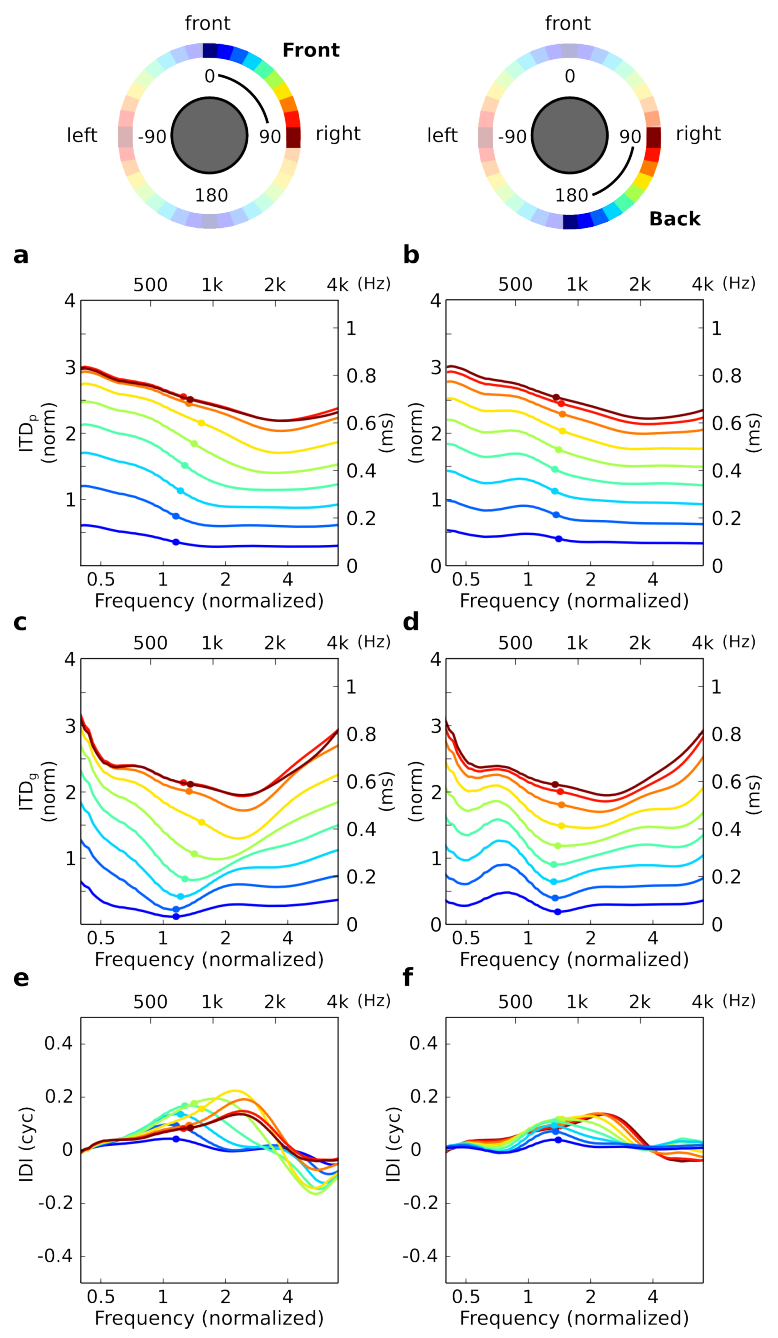

b

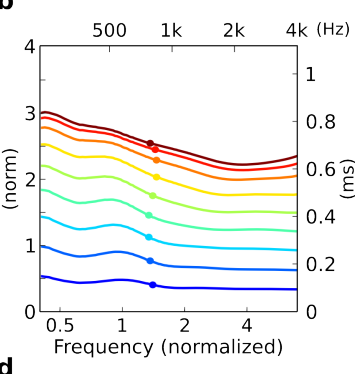

f

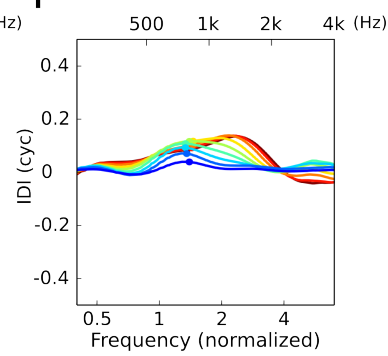

Figure 7: Averages over the whole population of normalized $\operatorname{ITD}_{\mathrm{p}}(\mathrm{a}, \mathrm{b}), \operatorname{ITD}_{\mathrm{g}}(\mathrm{c}, \mathrm{d})$, and IDI (e,f) for horizontal plane positions as a function of frequency. Top part of the figure depicts the lines color codes (positive azimuths, separated by $10^{\circ}$ ). Blue lines are more medial positions, and red more eccentric. Left column (a,c,e) displays data from the front positions, right column $(\mathrm{b}, \mathrm{d}, \mathrm{f})$ from the back positions. Dots overlaid on the line plot represent the position of the transition frequency.

359 the "transition frequency" introduced above (data not shown).

For all positions, we find that the IDI is close to zero for lower frequencies. It then 361 positively increases in low frequencies (below $2-3 \mathrm{kHz}$ ) and then drops to negative values for 362 higher frequencies (above about $4 \mathrm{kHz}$ ). Therefore, generally the fine-structure ITD is higher 
363 than the envelope ITD for frequencies between 1 and $3 \mathrm{kHz}$, and smaller in high frequencies

364 (around 4kHz). While both ITDs are monotonically increasing functions of eccentricity 365 (Figure 7a-b, c-d), the relationship is more complex for IDI. In addition, as noted in the 366 above statistical analyses, the frequency-dependence of ITD is different for front and back 367 positions (Figure 7e,f), which is potentially a cue to disambiguate between them.

\section{V. FREQUENCY-DEPENDENCE OF ITD IN ANIMALS}

369 In many animal species, neurons tuned to ITD have been identified, e.g. in the Medial 370 Superior Olive (MSO) or Inferior Colliculus (IC) of mammals (Yin and Chan, 1990). A 371 recent debate has emphasized the importance of the natural distribution of binaural cues in 372 our understanding of electrophysiological data (Grothe et al., 2010). In this context, and 373 more generally in neurophysiological studies of sound localization, binaural cues (ITD and 374 Interaural Intensity Differences, IID) have been measured for a number of animal species, 375 including mammals, but also birds and reptiles.

376 As seen in humans, a strong dependence on morphological features of the animals is 377 found in many instances. For example, the owl's facial ruff (Campenhausen and Wagner, 378 2006), or the cat's pinnae (Tollin and Koka, 2009) increase the magnitude of ITD at a given 379 position. However, the variation of ITD with frequency has received little attention until 380 recently (Benichoux et al., 2015). We applied the same analysis as above to measured HRTFs 381 of different animal species.

\section{A. Animal HRTF recordings}

383 We measured the HRTFs of six taxidermist animal models, using the same setup as 384 for the human recordings (LISTEN-V2, see Table I): rat, rabbit, guinea pig, chinchilla, 385 cat and macaque. All animals had their ear canals obstructed by the taxidermy, which 386 means that recordings are taken in a blocked-meatus configuration. Animal models were 387 chosen according to the well-preserved quality of their pinnae. We previously showed that 
HRTFs measured on taxidermist models agree closely with acoustical simulations based on

389 3D models of the animal with rigid boundaries (Rebillat et al., 2014).

390 In general, animal models were in a natural-looking position, in which the head of the

391 animal is not aligned with the body. Therefore, the coordinate system is rotated so that

392 the head points in the $0^{\circ}$ direction. This is achieved by computing the head angle relative

393 to the body: the azimuth that minimizes the magnitude of the low-frequency ITD value.

394 Head angles were generally non-zero for all models (rat $10^{\circ}$, rabbit $-10^{\circ}$, guinea pig $20^{\circ}$, 395 chinchilla $-20^{\circ}$, cat $-55^{\circ}$, macaque $-30^{\circ}$ ). It should also be noted that the interaural distance 396 of the rabbit, guinea pig and macaque models in the present study are noticeably smaller 397 than those of animals of the same species whose recordings are reported in the literature 398 (see Table II).

\section{B. Frequency-dependent ITD in animals}

A difference between low- and high-frequency ITD in animals has been previously shown

401 in a number of animal species: in the rat (Koka et al., 2008), rabbit (Kim et al., 2010), guinea 402 pig (Greene et al., 2014), chinchilla (Lupo et al., 2011; Koka et al., 2011), cat (Roth et al., 403 1980; Tollin and Koka, 2009) and macaque monkey (Spezio et al., 2000). Yet, only very 404 few studies reported the frequency-dependent ITD curves for many azimuth positions. The 405 phase ITD for all animal models and frequencies between 350 and $3000 \mathrm{~Hz}$ is reported on 406 Fig. 8, for positions in the horizontal plane. Consistent with physical acoustics, and the 407 above results in humans, the phase ITD is frequency-dependent in all species.

408 Because humans are bipeds, no part of the body normally finds itself on the way between 409 the source and the ears. In many quadrupeds, for example in the cat (Fig. 8e), sounds 410 coming from the back can be reflected or scattered by the body before reaching the head 411 and ears. This morphological asymmetry results in large differences between the frequency412 dependent ITDs of sources in the front (solid curves) and in the back (dashed). Thus in 413 principle, front and back positions can be distinguished on the basis of the ITD at different 


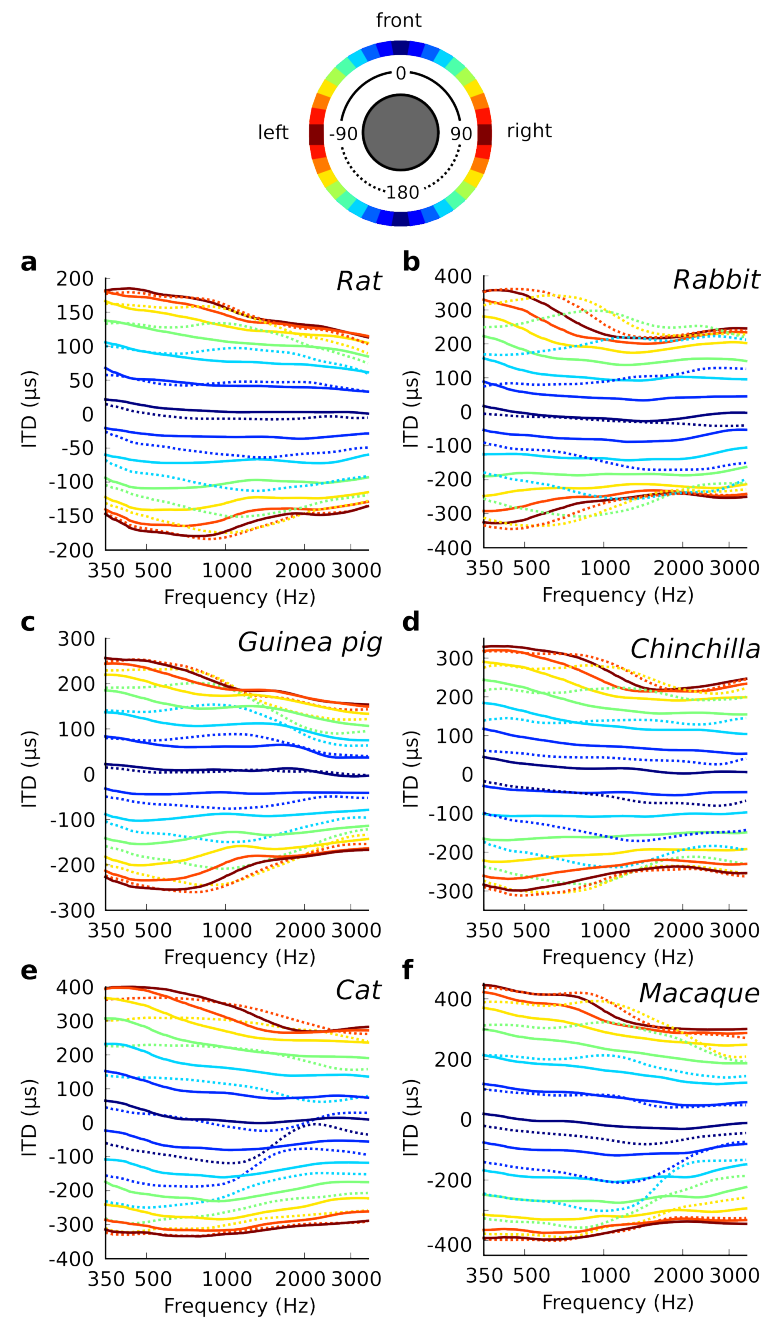

Figure 8: Animal ITDs in the horizontal plane, for 24 positions around the head (separated by $15^{\circ}$ ). Top: line color code; front positions, solid lines; back, dashed lines. (a) Rabbit, (b) Guinea pig, (c) Chinchilla, (d) Cat, (e) Rat, (f) Macaque.

414 frequencies. Similarly, it was shown using acoustical measures and simulations that the 415 posture of the animal influences the frequency-dependent pattern of ITD (Rebillat et al., 416 2014). 

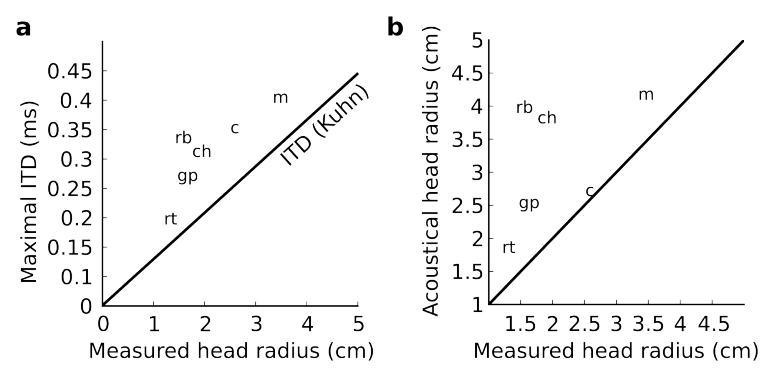

Figure 9: Comparison of measured ITD range with anatomy. (a) Maximal measured low-frequency ITD as a function of half the interaural distance measured on the taxidermist models. Predictions are shown for Kuhn's formula (solid). (b) Acoustical head radius estimated with Woodworth's formula (see Text) vs. half the interaural distance measured on the taxidermist models (dashed line: diagonal). Legend: rb: rabbit; ch: chinchilla; gp: guinea pig; m: macaque; rt: rat; c: cat.

\section{Estimating animal ITD from head size}

418 For electrophysiological studies, a way to estimate the maximal ITD for a given animal 419 species is to measure the interaural distance and then use one of Woodworth's (Eq. 2) or 420 Kuhn's formula (Eq. 3). The maximal ITD is thus obtained in low frequencies (via Eq. 3) 421 for the most eccentric position, that is $\mathrm{ITD}_{\max }=3 a / c$. However, in some species there is 422 evidence that this method yields an underestimation of the maximal ITD (see, e.g. (Tollin 423 and Koka, 2009) in the cat). "In Fig. 9a, the maximal ITD measured in the horizontal 424 plane is reported as a function of the half interaural distance of the animal, measured as the 425 half distance between the entrances of the (occluded) ear canals. In all cases, the maximal 426 ITD is well correlated with the physical head size, but substantially larger than predicted 427 using the Kuhn formula. We further computed the acoustical head radius for each animal 428 model, as we did in humans (see Section III). Consistent with previous observations (e.g. 429 (Tollin and Koka, 2009)), the acoustical head radius of animals is substantially larger than 430 their physical "head radius" (the half interaural distance of the animal model, Fig. 9b).

431 Overall, our animal results suggest that the maximal ITD should not be estimated from 
432 a crude measure of the morphology of the animal (here interaural distance), because this

433 leads to a systematic underestimation of the magnitude of ITD for all species.

\section{VI. SUMMARY AND DISCUSSION}

\section{A. Summary}

436 In this paper, we have quantified the variation of ITD with frequency in humans and 437 animals, measured in anechoic space. First, we confirmed that ITD does vary significantly 438 with frequency, as predicted by a spherical head model (Kuhn, 1977) and mentioned in 439 previous studies (Wightman and Kistler, 1989). Specifically, maximal ITD values were 440 found to be about $800 \mu \mathrm{s}$ in low frequencies and $600 \mu \mathrm{s}$ in high frequencies. Therefore, the 441 low frequency ITD can be larger than the high frequency ITD by as much as $200 \mu$ s, which 442 is an order of magnitude larger than human JNDs in ITD discrimination tasks (of the order 443 of $20 \mu \mathrm{s}$ ), even for pure tones (Brughera et al., 2013) (10-40 $\mu \mathrm{s}$ for frequencies below 1250 $444 \mathrm{~Hz})$. The transition between low- and high-frequency ITDs occurs at frequencies between 445600 and $1800 \mathrm{~Hz}$, within the range where ITD is a dominant cue for localization in the 446 horizontal plane (Wightman and Kistler, 1992).

447 Additionally, we observe that the frequency-dependence of ITD does not exhibit sim448 ple spherical symmetries. In particular, symmetrical front and back (and up and down) 449 positions, both in humans and animals, have different frequency-dependent ITDs. The fre450 quency dependence of ITD provides, in addition to azimuth, a cue to elevation including 451 information about front versus back.

452 We also show that for multiple of source positions, ITD varies not only globally across 453 the spectrum, but also locally within the bandwidth of a single auditory filter. This causes 454 different ITDs for envelope and fine-structure, which can provide additional information 455 about the position of the sound source. Furthermore, those cues can be estimated from 456 binaural signal using cross-correlation. The difference in group and fine-structure ITD is 457 quantified by the interaural diffraction index (IDI). 


\section{B. Relation with psychoacoustical experiments}

459

460

461

462

463

464

465

466

467

468

469

470

471

472

473

474

475 is entirely realistic.

476

477

478 Furthermore, in a two-dimensional absolute localization task the mean error is about $5^{\circ}$ in

479 the frontal hemifield (for broadband noise bursts, Makous and Middlebrooks (1990)), which

480 corresponds to about $50 \mu \mathrm{s}$ ITD. As the ITD variation across the spectrum can reach 200

$481 \mu$ s for some positions, systematic frequency-dependent errors should be observed if the ITD

482 variation were discarded.

483 Together, these studies suggest that human subjects can detect small ITD changes in 484 tones when they are presented in isolation, but they cannot detect them when they are 
485 embedded in a complex sound, as long the average ITD is unchanged. This is consistent 486 with the notion that source location is inferred from the pattern of ITD, but that only that 487 inferred location, rather than the acoustical cues, is available to conscious perception and 488 behavior, and in particular is used in discrimination tasks. Thus, two sounds with different 489 patterns of frequency-dependent ITD are indistinguishable if they yield the same estimated 490 location. This is consistent with other aspects of binaural hearing. In particular, it has been 491 shown that the sensitivity to interaural intensity differences (IID) is substantially degraded 492 when the use of intracranial position as a cue is eliminated by roving the the ITD of the 493 stimuli (Bernstein, 2004).

494 A possible experiment to determine whether ITD information is discarded in estimating

495 the location of the source is to include judgements of the position of sounds with different 496 frequency contents. For example, localization performance could be tested as in measured 497 HRTFs with linear phase HRTFs, but with band-pass filtered noises in different frequency 498 regions. If the frequency-dependence of ITD is discarded, then results should be identical 499 in the two conditions (provided the ITD of linear phase HRTFs is adjusted). On the other 500 hand, if the variation of ITD is indeed taken into account to estimate source position, we 501 should observe systematic errors depending on frequency and position.

\section{Binaural coherence}

503 Binaural coherence is defined as the maximal value of the cross-correlation of monaural 504 signals (Gabriel and Colburn, 1981). Humans are very sensitive to small changes in bin505 aural coherence, usually modeled by adding a small amount of independent noise at each 506 ear (usually below 3-4\% for noise (Gabriel and Colburn, 1981)). In HRTF recordings, bin507 aural coherence is found to be mainly affected by the amount reverberation in the room: 508 binaural coherence is very high in anechoic environments, and dramatically goes down as 509 the environment gets more reverberant (Hartmann et al., 2005). It can be argued that the 510 effect of the variation of ITD within an auditory filter is a decreased coherence (Constan 
511 and Hartmann, 2003). Yet, in anechoic conditions this effect remains marginal, especially

512 for the narrow bands of noise resulting from filtering by the auditory periphery (less than $5130.1 \%$ (Constan and Hartmann, 2003)).

514 We argue here for a different interpretation of the frequency-dependence of ITD. De515 coherence due to reverberation is intrinsically non-deterministic: different wavefronts reach 516 the listener at different times depending on the unknown geometry of the room. On the 517 other hand, we have shown above that the frequency-dependence of ITD has a fully deter518 ministic effect: envelope and fine-structure ITD cues are affected in a way that is predicted 519 by the morphology of the subject. Decoherence, insofar as it is non-deterministic, objec520 tively makes the task of recovering the ITD from the cross-correlation function harder. It 521 is unclear, however, why imposing different ITDs in the envelope and fine structure of the 522 monaural signals would make the recovering of ITDs harder, because it is fully deterministic.

523 Therefore, we argue that the variation of ITD in small frequency bands is best thought of as 524 imposing different ITDs in the envelope and fine-structure of monaural inputs, rather than 525 as causing binaural decoherence, as imposed by adding independent white noise to monaural 526 inputs.

\section{D. Signal processing of binaural sounds}

528 Our results are relevant to two classes of signal processing applications: reproduction 529 of binaural sounds and sound localization algorithms. The large variation of ITD with 530 frequency suggests that it is important for proper reproduction of binaural sounds. However, 531 it could be that humans can adapt to non-natural ITD patterns, as they do to spectral cues 532 (Wanrooij and Opstal, 2005). In either case, we note that replacing frequency-dependent 533 ITDs with fixed ITDs removes some potential cues to elevation.

534 State-of-the-art sound localization algorithms using HRTF-filtered inputs do use the 535 frequency-dependence of ITD to estimate source location. In the algorithm described by 536 May et al. (2011), sounds are divided into frequency bands, and position is estimated with a 
537 maximum likelihood approach from the overall ITDs in these bands. Because ITD likelihood

538 for each position is measured with KEMAR HRTFs, this algorithm uses the variation of ITD

539 across channels. However, it does not use the variation of ITD within channels.

540 Other algorithms use HRTF data with the within-channel ITD variations preserved,

541 (Durkovic et al., 2011; Macdonald, 2008) and were shown to perform well in realistic condi-

542 tions. In each frequency band, monaural signals are convolved with the contralateral HRTF

543 of a candidate source position (i.e., left signal with right HRTF), and the position giving the

544 highest cross-correlation is picked. A spiking neural model relying on similar ideas was also 545 previously presented (Goodman and Brette, 2010): it used cross-correlation, biophysically 546 modeled with coincidence detection between spike trains, and performed better when the 547 variation of ITD within channel was taken into account.

\section{E. Electrophysiology}

549 The firing rates of neurons in several auditory brainstem nuclei, in particular the me550 dial superior olive (MSO) and inferior colliculus (IC) of mammals, is sensitive to the ITD 551 of binaural sounds (Grothe et al., 2010). Similar to humans, we have shown that ITD is

552 frequency-dependent in animals, in the frequency range where it is used for sound localiza553 tion (Fig. 8). Furthermore, we showed that asymmetries in this frequency-dependence exist 554 between front and back positions, presumably due to reflections on the back of the animals 555 (Fig. 8). Finally, we have noted that the maximal ITD is generally larger than when esti556 mated from simple morphological considerations (Fig. 9). All these observations should be 557 taken into account when interpreting electrophysiological measurements.

558 In the physiological literature, two types of frequency-dependent properties have been 559 discussed (Grothe et al., 2010; Day and Semple, 2011; Benichoux et al., 2015). The preferred 560 ITD of binaural neurons, i.e., the ITD that elicits the largest firing rate, depends on their 561 preferred frequency: at the level of the population those quantities are inversely correlated. 562 This observation has been seen as a challenge to the mainstream theory, according to which 
563 neurons are tuned to the ITD of particular source locations, which should cover all possible

564 locations independently of the frequency band (Grothe et al., 2010). In our animal mea-

565 surements, ITD is also larger in lower frequencies than in high frequencies — although to a

566 smaller extent than in electrophysiological recordings. An additional contribution to large

567 low-frequency ITDs in animals is early reflections on the ground, which produce arbitrarily

568 large ITDs in low frequencies (Gourevitch and Brette, 2012).

569 Many binaural neurons also display a second type of frequency-dependence: for a given 570 neuron, the preferred ITD depends on the frequency of the sound (Day and Semple, 2011).

571 We have shown that ITD varies also with frequency within an auditory filter, which provides 572 a potential ecological explanation of this variation (Benichoux et al., 2015). The present 573 analysis suggests that cells with frequency-dependent best delays should be differentially 574 sensitive to envelope and fine-structure delays.

\section{Acknowledgments}

576 This work was supported by the European Research Council (ERC StG 240132). We 577 thank the Museum d'Histoire Naturelle de la Ville de Paris for lending the taxidermist 578 animal models, as well as Olivier Warusfel at IRCAM (Paris) for the anechoic chamber.

\section{References}

580 Aaronson, N. L. and Hartmann, W. M. (2014). "Testing, correcting, and extending the

581 Woodworth model for interaural time difference", The Journal of the Acoustical Society of 582 America 135, 817-823.

583 Algazi, V., Duda, R., Thompson, D., and Avendano, C. (2001a). "The CIPIC HRTF 584 database", in Proceedings of the IEEE Worshop on the Applications of Signal Processing 585 to Audio and Acoustics, 99-102.

586 Algazi, V. R., Avendano, C., and Duda, R. O. (2001b). "Estimation of a Spherical-Head 587 Model from Anthropometry", Journal of the Audio Engineering Society 49, 472-479. 
588 ARI (2010). "HRTF Database http://www.kfs.oeaw.ac.at/content/view/608/606/lang,8859589 1/ (date last viewed 13/8/2015)", .

590 Benichoux, V., Fontaine, B., Franken, T. P., Karino, S., Joris, P. X., and Brette, R. (2015).

591 "Neural tuning matches frequency-dependent time differences between the ears", eLife 4, $592 \mathrm{e} 06072$.

593 Bernstein, L. R. (2004). "Sensitivity to interaural intensitive disparities: listeners' use of 594 potential cues", The Journal of the Acoustical Society of America 115, 3156-3160.

595 Brughera, A., Dunai, L., and Hartmann, W. M. (2013). "Human interaural time difference 596 thresholds for sine tones: The high-frequency limit", The Journal of the Acoustical Society 597 of America 133, 2839-2855.

598 Bruneau, M. (2010). Fundamentals of acoustics (Wiley-ISTE, London, 636 pages).

599 Cai, T., Rakerd, B., and Hartmann, W. M. (2015). "Computing interaural differences 600 through finite element modeling of idealized human heads", The Journal of the Acousti601 cal Society of America 138, 1549-1560, URL http://scitation.aip.org/content/asa/ 602 journal/jasa/138/3/10.1121/1.4927491.

603 Campenhausen, M. v. and Wagner, H. (2006). "Influence of the facial ruff on the sound604 receiving characteristics of the barn owl's ears", Journal of Comparative Physiology A 192, $6051073-1082$.

606 Constan, Z. and Hartmann, W. (2003). "On the detection of dispersion in the head-related 607 transfer function", The Journal of the Acoustical Society of America 114, 998-1008.

608 Day, M. L. and Semple, M. N. (2011). "Frequency-dependent interaural delays in the medial 609 superior olive: implications for interaural cochlear delays", Journal of Neurophysiology 106, $6101985-1999$.

611 Duda, R. and Martens, W. (1998). "Range dependence of the response of a spherical head 612 model", The Journal of the Acoustical Society of America 104, 3048-3058.

613 Duda, R. O., Avendano, C., and Algazi, V. R. (1999). "An adaptable ellipsoidal head model 614 for the interaural time difference", in Proceedings of the IEEE International Conference on 615 Acoustics, Speech, and Signal Processing, 965-968. 
616 Durkovic, M., Habigt, T., Rothbucher, M., and Diepold, K. (2011). "Low latency localiza617 tion of multiple sound sources in reverberant environments", The Journal of the Acoustical 618 Society of America 130, EL392-EL398.

619 Gabriel, K. J. and Colburn, H. S. (1981). "Interaural correlation discrimination: I. Band620 width and level dependence", The Journal of the Acoustical Society of America 69, 13946211401.

622 Glasberg, B. R. and Moore, B. C. (1990). "Derivation of auditory filter shapes from notched623 noise data", Hearing research 47, 103-138.

624 Goodman, D. F. M. and Brette, R. (2010). "Spike-Timing-Based Computation in Sound 625 Localization", PLoS Comput Biol 6, e1000993.

626 Gourevitch, B. and Brette, R. (2012). "The impact of early reflections on binaural cues", 627 The Journal of the Acoustical Society of America 132, 9-27.

628 Greene, N. T., Anbuhl, K. L., Williams, W., and Tollin, D. J. (2014). "The acoustical cues 629 to sound location in the guinea pig (Cavia porcellus)", Hearing Research 316C, 1-15.

630 Grothe, B., Pecka, M., and McAlpine, D. (2010). "Mechanisms of sound localization in 631 mammals", Physiological Reviews 90, 983-1012.

632 Hartmann, W. and Wittenberg, A. (1996). "On the externalization of sound images", The 633 Journal of the Acoustical Society of America 99, 3678-3688.

634 Hartmann, W. M., Rakerd, B., and Koller, A. (2005). "Binaural coherence in rooms", Acta 635 acustica united with acustica 91, 451-462.

636 Kim, D. O., Bishop, B., and Kuwada, S. (2010). "Acoustic Cues for Sound Source Dis637 tance and Azimuth in Rabbits, a Racquetball and a Rigid Spherical Model", Journal of the 638 Association for Research in Otolaryngology 11, 541-557.

639 Kistler, D. J. and Wightman, F. L. (1992). "A model of Head-Related Transfer Functions 640 based on Principal Components Analysis and Minimum-Phase Reconstruction", Journal of 641 the Acoustical Society of America 91, 1637-1647.

642 Koka, K., Jones, H. G., Thornton, J. L., Lupo, J. E., and Tollin, D. J. (2011). "Sound pres643 sure transformations by the head and pinnae of the adult Chinchilla (Chinchilla lanigera)", 
644 Hearing Research 272, 135-147.

645 Koka, K., Read, H. L., and Tollin, D. J. (2008). "The acoustical cues to sound location in

646 the rat: Measurements of directional transfer functions", Journal of the Acoustical Society

647 of America 123, 4297-4309.

648 Kuhn, G. (1977). "Model for interaural time difference in azimuthal plane", Journal of the 649 Acoustical Society of America 62, 157-167.

650 Kulkarni, A., Isabelle, S., and Colburn, H. (1999). "Sensitivity of human subjects to head651 related transfer-function phase spectra", Journal of the Acoustical Society of America 105, $6522821-2840$.

653 Luling, H., Siveke, I., Grothe, B., and Leibold, C. (2011). "Frequency-Invariant Represen654 tation of Interaural Time Differences in Mammals", PLoS Comput Biol 7, e1002013.

655 Lupo, J. E., Koka, K., Thornton, J. L., and Tollin, D. J. (2011). "The effects of experimen656 tally induced conductive hearing loss on spectral and temporal aspects of sound transmission 657 through the ear", Hearing Research 272, 30-41.

658 Macdonald, J. A. (2008). "A localization algorithm based on head-related transfer func659 tions", The Journal of the Acoustical Society of America 123, 4290-4296.

660 Makous, J. C. and Middlebrooks, J. C. (1990). "Two-dimensional sound localization by 661 human listeners", The Journal of the Acoustical Society of America 87, 2188-2200.

662 Marple Jr, S. L. (1999). "Estimating group delay and phase delay via discrete-time "ana663 lytic" cross-correlation", Signal Processing, IEEE Transactions on 47, 2604-2607.

664 May, T., van de Par, S., and Kohlrausch, A. (2011). "A Probabilistic Model for Robust Lo665 calization Based on a Binaural Auditory Front-End", IEEE Transactions on Audio, Speech, 666 and Language Processing 19, 1-13.

667 Middlebrooks, J. C. (1999). "Individual differences in external-ear transfer functions reduced 668 by scaling in frequency", The Journal of the Acoustical Society of America 106, 1480-1492. 669 Mills, A. W. (1958). "On the minimum audible angle", The Journal of the Acoustical Society 670 of America 30, 237.

671 Papadatos, N. (1995). "Maximum variance of order statistics", Annals of the Institute of 
673 Rayleigh, L. and Lodge, A. (1904). "On the Acoustic Shadow of a Sphere. With an Ap674 pendix, Giving the Values of Legendre's Functions from P0 to P20 at Intervals of 5 Degrees", 675 Philosophical Transactions of the Royal Society of London. Series A, Containing Papers of 676 a Mathematical or Physical Character 203, 87-110.

677 Rebillat, M., Benichoux, V., Otani, M., Keriven, R., and Brette, R. (2014). "Estimation 678 of the low-frequency components of the head-related transfer functions of animals from 679 photographs", The Journal of the Acoustical Society of America 135, 2534-2544.

680 Roth, G., Kochhar, R., and Hind, J. (1980). "Inter-aural Time Differences - Implications 681 regarding the neurophysiology of sound localization", Journal of the Acoustical Society of 682 America 68, 1643-1651.

683 Spezio, M., Keller, C., Marrocco, R., and Takahashi, T. (2000). "Head-related transfer 684 functions of the Rhesus monkey", Hearing Research 144, 73-88.

685 Tollin, D. J. and Koka, K. (2009). "Postnatal development of sound pressure transformations 686 by the head and pinnae of the cat: binaural characteristics", The Journal of the Acoustical 687 Society of America 126, 3125.

688 Wanrooij, M. M. V. and Opstal, A. J. V. (2005). "Relearning Sound Localization with a 689 New Ear", The Journal of Neuroscience 25, 5413-5424.

690 Warusfel, O. (2002). "LISTEN HRTF database, http://recherche.ircam.fr/equipes/salles/listen 691 (date last viewed 13/8/2015)", .

692 Wightman, F. L. and Kistler, D. J. (1989). "Headphone Simulation of Free-Field Listening. 693 1. Stimulus Synthesis", Journal of the Acoustical Society of America 85, 858-867.

694 Wightman, F. L. and Kistler, D. J. (1992). "The Dominant Role of Low-frequency Inter695 aural Time Differences inf Sound Localization", Journal of the Acoustical Society of America 696 91, 1648-1661.

697 Yin, T. C. and Chan, J. C. (1990). "Interaural time sensitivity in medial superior olive of 698 cat", Journal of Neurophysiology 64, 465-488. 


\begin{tabular}{lrrrrc} 
Database & $N_{s}$ & $\Delta \theta$ & $\Delta \phi$ & $N_{\text {sub }}$ & Room type \\
\hline \hline LISTEN-V1 & 8192 & $15^{\circ}$ & $\simeq 15^{\circ}$ & 49 & Anechoic \\
LISTEN-V2 & 8192 & $5^{\circ}$ & $\simeq 15^{\circ}$ & 35 & Anechoic \\
CIPIC & 200 & $\simeq 10^{\circ}$ & $5.6^{\circ}$ & 36 & Anechoic \\
ARI & 2400 & $5^{\circ}$ & $10^{\circ}$ & 10 & Semi-anechoic
\end{tabular}

Table I: Overview of the different human HRTF databases used in this study. For each database, the sampling frequency is $44.1 \mathrm{kHz} . N_{s}$ : length of the head-related impulse responses in samples. $\Delta \theta, \Delta \phi$ : approximate spatial resolution in azimuth and elevation. $N_{s u b}$ : number of subjects from each database included in the present study. The LISTEN database consists of the 49 subjects freely available on the IRCAM website (LISTEN-V1) and of 35 subjects measured later with an increased spatial resolution in azimuth (LISTEN-V2). Measurements for the ARI database have been performed under semi-anechoic conditions and because of measurement artifacts, only 10 subjects have been retained and the spatial resolution in elevation has been decreased to $10^{\circ}$. 
Max ITD Interaural distance

Animal LF HF Tax. models Reported Acoustical

\begin{tabular}{lcccc}
\hline \hline Rat & $165 \mu \mathrm{s} 134 \mu \mathrm{s}$ & $2.7 \mathrm{~cm}$ & $2.96 \mathrm{~cm}^{1}$ & $3.78 \mathrm{~cm}$ \\
Rabbit & $319 \mu \mathrm{s} 246 \mu \mathrm{s}$ & $3.2 \mathrm{~cm}$ & $5.6 \mathrm{~cm}^{2}$ & $8.02 \mathrm{~cm}$ \\
Guinea pig $242 \mu \mathrm{s} 184 \mu \mathrm{s}$ & $3.35 \mathrm{~cm}$ & $4.94 \mathrm{~cm}^{3}$ & $5.02 \mathrm{~cm}$ \\
Chinchilla & $293 \mu \mathrm{s} 240 \mu \mathrm{s}$ & $3.9 \mathrm{~cm}$ & $3.6 \mathrm{~cm}^{4}$ & $7.68 \mathrm{~cm}$ \\
Cat & $335 \mu \mathrm{s} 276 \mu \mathrm{s}$ & $5.2 \mathrm{~cm}$ & $5.6 \mathrm{~cm}^{5}$ & $5.44 \mathrm{~cm}$ \\
Macaque & $393 \mu \mathrm{s} 310 \mu \mathrm{s}$ & $7.0 \mathrm{~cm}$ & $10.4 \mathrm{~cm}^{6}$ & $8.36 \mathrm{~cm}$
\end{tabular}

Table II: Overview of the animal ITD data. Maximal ITDs measured in low and high frequencies for the animal HRTFs. Interaural distances are the distances measured between the ear canal entrances of the taxidermized models (Tax. models), or the value as reported in previous studies (Reported), or twice the acoustical head radius (Acoustical, estimated from ITDs). References: ${ }^{1}$ (Koka et al., 2008), ${ }^{2}$ (Kim et al., 2010), ${ }^{3}$ (Greene et al., 2014), ${ }^{4}$ (Lupo et al., 2011), ${ }^{5}$ (Roth et al., 1980), ${ }^{6}$ (Spezio et al., 2000) . 


\section{List of Figures}

700 Figure 1 Frequency-dependence of ITD. (a) ITD measured with pure tones of varying

701

702

703

704

705

706

707 frequency for different source positions on a human manikin (replotted from Kuhn (1977)). (b) ITD computed for a spherical head model with head radius $9.3 \mathrm{~cm}$. (c) Propagation of a planar sound wave with an acoustically transparent head. The additional pathlength to the contralateral ear (thick line) is a sine function of the azimuth angle $\theta$. (d) Propagation of a high frequence planar sound wave diffracted by a sphere. The additional path to the contralateral ear is the thick line. . . . . . . . . . . . .

708 Figure 2 Propagation time of a planar sound wave in the presence of a sphere, relative to the propagation time in free field, for tone frequencies $114.5 \mathrm{~Hz}$ (a) and $1145 \mathrm{~Hz}$ (b). Propagation time in free field (no head) is shown on top. Negative values (lighter shades) indicate regions where phase is leading, and positive values (darker shades) indicate regions where the sound phase is

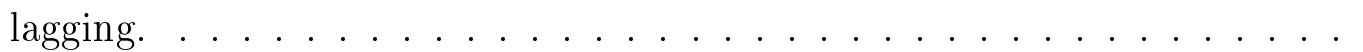


714 Figure 3 Frequency dependence of ITD in human subjects. (a) Inter-individual average

715

716

717

718

719

720

721

722

723

724

725

726 normalized ITD in high frequency as a function of azimuth $\pm 1 / 2$ s.d. (shaded area). Black line indicates the theoretical value from the Woodworth model (Eq. 2). Corresponding ITD values for a head radius of $9.5 \mathrm{~cm}$ are shown on the right of panel b. (b) Average normalized ITD in low frequency $\pm 1 / 2$ s.d. (shaded area), black line indicates the theoretical value from Kuhn's formula (Eq. 3). (c) Average normalized ITD (black lines) as a function of frequency for seven source positions (shaded area: $\pm 1 / 2$ s.d.). (d) Azimuth $\theta$ and elevation $\phi$ are defined in a standard vertical-polar coordinate system (see text). (e) Difference between high- and low-frequency normalized ITD as a function of elevation and azimuth. Physical ITD is calculated for a head radius of $9.5 \mathrm{~cm}$. (f) Same as (e) for the spherical model. Normalized units correspond to a head radius of $9.5 \mathrm{~cm}$.

727 Figure 4 Maximal ITD and transition frequency in human subjects. (a) Maximal ITD 728 across subjects as a function of frequency. (b) Azimuth (top) and elevation (bottom) where ITD is maximal as a function of frequency Shaded areas of 730 (a) and (b) are the mean $\pm 1 / 2$ s.d. (c) Transition frequency (see text) as a 731 732 function of azimuth and elevation in humans (left) and in the spherical model (right). Normalized units converted assuming a head radius of $9.5 \mathrm{~cm}$. . .

733 Figure 5 Variation of ITD within single auditory filters. (a) Schematics of the global variation of ITD across different auditory filters. (b) Schematics of the variation of ITD within a single auditory filter. (c) Proportion of positions and center frequencies where ITD variation within a single channel is smaller than a specified value. Lines are averages across population, \pm s.d.. Channel width is either 1 ERB or $1 / 3$ octave. (d) Maximal ITD variation within single channels, as a function of azimuth and elevation, with ERB-wide channels. 
740 Figure 6 Envelope and fine-structure ITD. (a) The IPD for one position is unwrapped and an affine fit is taken locally around $f_{0}$. The intercept of the fit is the IDI and the slope the group ITD. (b) When the IDI is zero, the delay is frequencyindependent and both envelope and fine-structure are delayed by the ITDg (bottom, black signal). When IDI is non zero, the fine-structure undergoes an additional phase shift equal to the IDI (bottom, grey signal). (c) Simulation: white noise is passed through HRTF filters for one position (spherical head model, azimuth $=70^{\circ}$ ). The resulting signals are then fed into gammatone filterbanks. The responses in the two banks are then cross-correlated, and the result is separated in envelope and fine-structure components. The time lag of the maximum of the cross-correlation is the phase ITD, and that of the maximum of the envelope of the cross-correlation is the envelope ITD (see text). (d) Results of estimating phase ITD, ITD , from the IPD (plain line), and from simulations (mean: dots, shaded area: 95\% confidence interval).

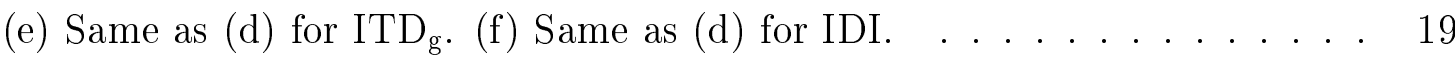

755 Figure 7 Averages over the whole population of normalized $\operatorname{ITD}_{\mathrm{p}}(\mathrm{a}, \mathrm{b}), \operatorname{ITD}_{\mathrm{g}}(\mathrm{c}, \mathrm{d})$, 756 and IDI (e,f) for horizontal plane positions as a function of frequency. Top part of the figure depicts the lines color codes (positive azimuths, separated by $\left.10^{\circ}\right)$. Blue lines are more medial positions, and red more eccentric. Left column (a,c,e) displays data from the front positions, right column (b,d,f) from the back positions. Dots overlaid on the line plot represent the position

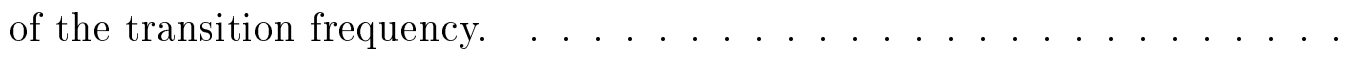

762 Figure 8 Animal ITDs in the horizontal plane, for 24 positions around the head (sepa763 rated by $15^{\circ}$ ). Top: line color code; front positions, solid lines; back, dashed lines. (a) Rabbit, (b) Guinea pig, (c) Chinchilla, (d) Cat, (e) Rat, (f) Macaque. 24 
765 Figure 9 Comparison of measured ITD range with anatomy. (a) Maximal measured 766 low-frequency ITD as a function of half the interaural distance measured on 767 the taxidermist models. Predictions are shown for Kuhn's formula (solid). (b) 768 Acoustical head radius estimated with Woodworth's formula (see Text) vs. 769 half the interaural distance measured on the taxidermist models (dashed line: 770 diagonal). Legend: rb: rabbit; ch: chinchilla; gp: guinea pig; m: macaque; 771

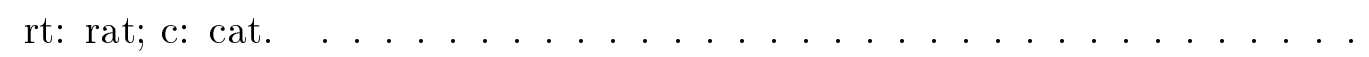

